\title{
A Semianalytical Approach to the Solution of Time-Fractional Navier-Stokes Equation
}

\author{
Zeeshan Ali $\mathbb{D}^{1},{ }^{1}$ Shayan Naseri Nia, ${ }^{1}$ Faranak Rabiei $\left(\mathbb{D},{ }^{1}\right.$ Kamal Shah $\left(\mathbb{D},{ }^{2,3}\right.$ \\ and Ming Kwang Tan $\left(\mathbb{D}^{1}\right.$ \\ ${ }^{1}$ School of Engineering, Monash University Malaysia, 47500 Selangor, Malaysia \\ ${ }^{2}$ Department of Mathematics, University of Malakand, Dir(L), 18000 Khyber Pakhtunkhwa, Pakistan \\ ${ }^{3}$ Department of Mathematics and General Sciences, Prince Sultan University, Saudi Arabia
}

Correspondence should be addressed to Zeeshan Ali; zeeshan.ali@monash.edu

Received 12 February 2021; Revised 19 April 2021; Accepted 26 June 2021; Published 19 July 2021

Academic Editor: ZEA Fellah

Copyright ( 2021 Zeeshan Ali et al. This is an open access article distributed under the Creative Commons Attribution License, which permits unrestricted use, distribution, and reproduction in any medium, provided the original work is properly cited.

\begin{abstract}
In this manuscript, a semianalytical solution of the time-fractional Navier-Stokes equation under Caputo fractional derivatives using Optimal Homotopy Asymptotic Method (OHAM) is proposed. The above-mentioned technique produces an accurate approximation of the desired solutions and hence is known as the semianalytical approach. The main advantage of OHAM is that it does not require any small perturbations, linearization, or discretization and many reductions of the computations. Here, the proposed approach's reliability and efficiency are demonstrated by two applications of one-dimensional motion of a viscous fluid in a tube governed by the flow field by converting them to time-fractional Navier-Stokes equations in cylindrical coordinates using fractional derivatives in the sense of Caputo. For the first problem, OHAM provides the exact solution, and for the second problem, it performs a highly accurate numerical approximation of the solution compare with the exact solution. The presented simulation results of OHAM comparison with analytical and numerical approaches reveal that the method is an efficient technique to simulate the solution of time-fractional types of Navier-Stokes equation.
\end{abstract}

\section{Introduction}

Partial differential equations (PDEs) are utilized to mathematically formulate and thus help solve physical and other problems involving functions of several variables, such as the propagation of sound or heat, electrostatics, fluid flow, elasticity, and electrodynamics. In fluid mechanics, the Navier-Stokes equation is a PDE that illustrates incompressible fluids' flow. This equation is a generalization of the equation developed to illustrate the flow of frictionless and incompressible fluids by Euler in the eighteenth century. In 1821, Navier added the viscosity (friction) element to make viscous fluids more realistic and complex. The British physicist and mathematician Stokes improved it during the middle of the nineteenth century, though complete solutions were achieved only for simple two-dimensional flows [1]. That is why the equation is called the Navier-Stokes equation. The mathematical model of the above-mentioned equation is given by:

$$
\frac{\partial \mathcal{U}(s, t)}{\partial t}+(\mathscr{U}(s, t) \cdot \nabla) \mathcal{U}(s, t)=-\frac{1}{\rho} \nabla \mathbf{p}+\vartheta \nabla^{2} \mathcal{U}(s, t),
$$

where $\mathcal{U}$ is the velocity, $t$ is the time, $\rho$ is the density, $s$ is the spatial variable, $\vartheta$ is the kinematics viscosity, $p$ is the pressure, and $\nabla$ denotes the gradient differential operator.

Fractional differential equations have proven to be a powerful tool for modeling real-world problems in the literature. It was noticed that time-fractional derivatives usually appear as infinitesimal generators of the time evolution when choosing a long-time scaling limit. Several essential phenomena in physics and polymer technology [2], electrical circuits [3], electrochemistry [4], electrodynamics 
of complex medium [5], control theory [6], thermodynamics [7], viscoelasticity [8], aerodynamics [9], capacitor theory [10], biology [11], blood flow [12], and fitting of experimental data [13], are well described by the aforesaid equations. Equation (1) can be converted to time-fractional derivative of order $\alpha \in(0,1]$, as given by:

$$
\frac{\partial^{\alpha} \mathcal{U}(s, t)}{\partial t^{\alpha}}+(\mathcal{U}(s, t) \cdot \nabla) \mathcal{U}(s, t)=-\frac{1}{\rho} \nabla \mathbf{p}+\vartheta \nabla^{2} \mathcal{U}(s, t) .
$$

Here, $\partial^{\alpha} / \partial t^{\alpha}$ is the Caputo fractional derivative. As the equation mentioned above is nonlinear, there is no known general method for resolving it. There are very few cases where it is possible to achieve the exact solution of Equation (2), making some predictions about the fluid's state and a simple arrangement considered for the flow pattern; for detail, see [14].

Analytical and numerical techniques are extensively utilized to solve nonlinear differential equations modeling physical phenomena. This is because the exact solutions of the above-mentioned equations are challenging to achieve. In recent decades, a new variety of perturbation methods have developed, which is loosely based on Poincare's homotopy applied in topology. Liao [15] introduced the Homotopy Analysis Method (HAM) in 1992. In 1998, He [16] followed Liao's work and developed the Homotopy Perturbation Method (HPM). Both methods have been successfully implemented to the problems, which exist in engineering and science fields. For example, Ganji and Rafei [17] solved nonlinear Hirota-Satsuma coupled Korteweg-De Vries equation by HPM. Lu and Liu [18] solved the Korteweg-De VriesBurgers equation via the help of HAM. Siddiqui et al. [19] utilized HPM and examined the irregular 2D flow of a viscous magnetohydrodynamics fluid within two parallel plates.

In [20], Marinca and Herisanu proposed a technique called OHAM. The benefit of the above-mentioned technique is in the built-in convergence criteria alike to HAM but extraflexible. The researchers have successfully implemented this approach to solving essential science problems and have also explained its reliability and effectiveness, for example, the dynamics of an electrical machine exhibiting nonlinear vibration [21], the oscillations of a particle that moves on a rotating parabola [22], the explicit solutions for some oscillators with discontinuities and a fractional power restoring force [23], and nonlinear equations arising in heat transfer [20], in an application to the steady flow of a fourth-grade fluid [24]. The above-mentioned technique is the HAM's modification, which is based on minimizing the residual error. In OHAM, the adjustment and control of the convergence region are provided conveniently.

In [14], Momani and Odibat considered unsteady onedimensional motion of a viscous fluid in a tube. The equations of motion which govern the flow field in the tube are the Navier-Stokes equations in cylindrical coordinates. They converted Equation (2) to the operator form as:

$$
\frac{\partial^{\alpha} \mathcal{U}(s, t)}{\partial t^{\alpha}}=\mathrm{P}+\vartheta\left(\frac{\partial^{2} \mathcal{U}(s, t)}{\partial s^{2}}+\frac{1}{s} \frac{\partial \mathcal{U}(s, t)}{\partial s}\right),
$$

where $0<\alpha \leq 1$ is the fractional order derivative and $P=-\partial \mathbf{p} / \rho \partial z$. For $\alpha=1$, we can get the standard NavierStokes equation. For the analytical solution, they have utilized the Adomian decomposition method. Lately, several powerful analytical techniques have been utilized to achieve the solution of Equation (3), such as the modified Laplace decomposition method [25], the $q$-homotopy analysis transform scheme [26], the new homotopy perturbation transform method [27], the iterative Elzaki transform method [28], the natural homotopy perturbation method [29], Elzaki transform with homotopy perturbation technique [30], and He's homotopy perturbation and variational iteration methods [31]. The aforementioned methods are called analytical, and no one has used the semianalytical technique for the solution of Equation (3) in the previous studies. Therefore, the objective of this manuscript is to present the semianalytical solution of Equation (3) by a semianalytical approach called OHAM.

The rest of the manuscript is structured as follows: in Section 2, we recall some definitions and properties of noninteger order operators. Section 3 is devoted to the basic formulation of OHAM. In Section 4, we apply the above-mentioned technique to time-fractional Navier-Stokes type of equations and discuss the method reliability through tables and plots. Section 5 is devoted to the conclusion.

\section{Definitions and Properties}

This section deals with some definitions and properties that are used in the manuscript.

Definition 1. A real function $\psi(t), t>0$ is supposed to be in the space $C_{\kappa}(\kappa>0)$ if it can express as $\psi(t)=t^{p} \psi_{1}(t)$ for certain $p>\kappa$ where $\psi_{1}(t) \in C[0, \infty)$, and it is supposed to be in the space $\mathrm{C}_{\kappa}^{m} \Leftrightarrow \psi^{m} \in \mathrm{C}_{\kappa}, m \in \mathbb{N}$.

Definition 2 (see [32]). The $\alpha \geq 0$ order integral operator for a function $\psi \in C_{\kappa}, \kappa \geq-1$ in the Riemann-Liouville sense is defined as:

$$
\mathbf{I}^{\alpha} \psi(t)=\frac{1}{\Gamma(\alpha)} \int_{0}^{t} \frac{\psi(\theta)}{(t-\theta)^{1-\alpha}} d \theta
$$

Let $\psi \in C_{\kappa}, \kappa \geq-1, \alpha, \gamma \geq 0$, and $\mu>-1$, then we have the properties [32] given by:

$$
\begin{aligned}
\mathbf{I}^{\alpha} \mathbf{I}^{\gamma} \psi(t) & =\mathbf{I}^{\alpha+\gamma} \psi(t), \\
\mathbf{I}^{\alpha} \mathbf{I}^{\gamma} \psi(t) & =\mathbf{I}^{\gamma} \mathbf{I}^{\alpha} \psi(t), \\
\mathbf{I}^{\alpha} t^{\mu} & =\frac{\Gamma(\mu+1)}{\Gamma(\mu+\alpha+1)} t^{\mu+\alpha} .
\end{aligned}
$$

Definition 3 (see [32]). The $\alpha>0$ order Caputo derivative operator for a function $\psi \in \mathrm{C}_{-1}^{m}, m \in \mathbb{N}$ is defined as:

$$
{ }^{c} \mathbf{D}^{\alpha} \psi(t)=\frac{1}{\Gamma(n-\alpha)} \int_{0}^{t} \frac{\psi^{(n)}(\theta)}{(t-\theta)^{\alpha-n+1}} d \theta, \quad t>0,
$$


where $[\alpha]$ is the integer part of $\alpha$ and $n=[\alpha]+1$. For $\psi$, $\phi \in \mathrm{C}_{\kappa}^{m}, \kappa \geq-1$, we have the properties [32] given by:

$$
\begin{aligned}
{ }^{c} \mathbf{D}^{\alpha}(a \psi(t)+b \phi(t)) & =a^{c} \mathbf{D}^{\alpha} \psi(t)+b^{c} \mathbf{D}^{\alpha} \phi(t), \quad a, b \in \mathrm{IR}, \\
{ }^{c} \mathbf{D}^{\alpha} \mathbf{I}^{\alpha} \psi(t) & =\psi(t), \\
\mathbf{I}^{\alpha}\left[{ }^{c} \mathbf{D}^{\alpha}\right] \psi(t) & =\psi(t)-\sum_{j=0}^{k-1} \psi^{(j)}(0) \frac{x_{j}}{j !} .
\end{aligned}
$$

\section{Basic Formulation of the OHAM}

We formulate OHAM for PDEs with boundary condition, in the steps given by [33]:

$\mathscr{L}(\mathscr{U}(s, t))+\mathscr{N}(\mathscr{U}(s, t))+\hbar(s, t)=0, \mathscr{B}\left(\mathscr{U}, \frac{\partial \mathscr{U}}{\partial t}\right)=0, \quad s \in \mathcal{Y}$,

where $\mathscr{L}$ and $\mathcal{N}$ are the linear and nonlinear operators, respectively. $\mathscr{U}(s, t)$ is an unknown function, the boundary operator denoted by $\mathscr{B}, s$ and $t$ denote spatial and time variables, respectively, $\hbar(s, t)$ is known function, and $r$ is the domain of the problem.

By OHAM, we construct the homotopy $\omega(s, t ; \wp)$ : $r \times J \longrightarrow R$, where $J=[0,1]$, which satisfies:

$$
\begin{aligned}
(1-\wp)\{\mathscr{L}(\omega(s, t ; \wp))+\hbar(s, t)\}= & \mathscr{H}(\wp)\{\mathscr{L}(\omega(s, t ; \wp)) \\
& +\mathscr{N}(\omega(s, t ; \wp))+\hbar(s, t)\},
\end{aligned}
$$

where $\wp \in J$ is an embedding parameter and $\mathscr{H}(\wp)$ is a nonzero auxiliary function for $\wp \neq 0$ and $\mathscr{H}(0)=0$. Equation (9) is to be the optimal homotopy equation. Obviously,

$$
\begin{aligned}
& \wp=0 \Rightarrow \mathscr{L}(\omega(s, t ; \wp))+\hbar(s, t)=0, \\
& \wp=1 \Rightarrow \mathscr{L}(\omega(s, t ; \wp))+\mathscr{N}(\omega(s, t ; \wp))+\hbar(s, t) .
\end{aligned}
$$

For $\wp=0$, we can obtain $\omega(s, t ; 0)=\mathscr{U}_{0}(s, t)$, and for $\wp=1$, we can get $\omega(s, t ; 1)=\mathscr{U}(s, t)$. Therefore, as $\wp$ extend from 0 to 1 , then $\omega(s, t ; \wp)$ moves from $\mathscr{U}_{0}(s, t)$ to $\mathscr{U}(s, t)$, where $\mathscr{U}_{0}(s, t)$ is got from Equation (9) for $\wp=0$ :

$$
\mathscr{L}\left(\mathscr{U}_{0}(s, t ; \wp)\right)+\hbar(s, t)=0, \mathscr{B}\left(\mathscr{U}_{0}, \frac{\partial \mathscr{U}_{0}}{\partial t}\right)=0 .
$$

Now, we take $\mathscr{H}(\wp)$, which is called auxiliary function, in the following form:

$$
\mathscr{H}(\wp)=\wp C_{1}+\wp^{2} C_{2}+\wp^{3} C_{3}+\cdots+\wp^{m} C_{m} .
$$

For the numerical solution, we utilize Taylor's series about $\wp$ and expand $\omega\left(\mathrm{s}, \mathrm{t} ; \wp, C_{p}\right)$ in the following way:

$$
\omega\left(s, t ; \wp, C_{p}\right)=\mathscr{U}_{0}(s, t)+\sum_{q-1}^{\infty} \mathcal{U}_{q}\left(s, t ; C_{p}\right) \wp^{q}, \quad p=1,2,3, \cdots
$$

Plugging Equation (13) in Equation (9) and equating the coefficient of like powers of $\wp$, we get the problem of zerothorder; given in (11), the problems of the first and second order are given by the Equations (14) and (15), respectively, and the general governing equations for $\mathcal{U}_{\mathrm{q}}(s, t)$ are given in Equation (16):

$$
\begin{aligned}
& \mathscr{L}\left(\mathcal{U}_{1}(s, t)\right)=C_{1} \mathcal{N}_{0}\left(\mathscr{U}_{1}(s, t)\right), \mathscr{B}\left(\mathcal{U}_{1}, \frac{\partial \mathcal{U}_{1}}{\partial t}\right)=0, \\
& \mathscr{L}\left(\mathscr{U}_{2}(s, t)\right)-\mathscr{L}\left(\mathscr{U}_{1}(s, t)\right)=C_{2} \mathcal{N}_{0}\left(\mathscr{U}_{0}(s, t)\right)+C_{1}\left[\mathscr{L}\left(\mathscr{U}_{1}(s, t)\right)+\mathcal{N}_{1}\left(\mathscr{U}_{0}(s, t), \mathscr{U}_{1}(s, t)\right)\right], \mathscr{B}\left(\mathscr{U}_{2}, \frac{\partial \mathscr{U}_{2}}{\partial t}\right)=0 \\
& \mathscr{L}\left(\mathcal{U}_{q}(s, t)\right)-\mathscr{L}\left(\mathcal{U}_{q-1}(s, t)\right)=C_{q} \mathcal{N}_{0}\left(\mathscr{U}_{0}(s, t)\right)+\sum_{p=1}^{q-1} C_{p}\left[\mathscr{L}\left(\mathcal{U}_{q-p}(s, t)\right)\right. \\
& \left.+\mathcal{N}_{q-p}\left(\mathcal{U}_{0}(s, t), \mathscr{U}_{1}(s, t), \mathscr{U}_{2}(s, t), \cdots, \mathscr{U}_{q-p}(s, t)\right)\right], \mathscr{B}\left(\mathscr{U}_{q}, \frac{\partial \mathcal{U}_{q}}{\partial t}\right), \quad q=2,3, \cdots,
\end{aligned}
$$


where $\mathcal{N}_{q-p}\left(\mathscr{U}_{0}(s, t), \mathscr{U}_{1}(s, t), \cdots, \mathscr{U}_{q-p}(s, t)\right)$ is the coefficient of $\wp^{q-p}$ in the expansion of $\mathcal{N}(\omega(s, t ; \wp))$ about the embedding parameter $\wp$.

$\mathcal{N}\left(\omega\left(s, t ; \wp, C_{p}\right)\right)=\mathcal{N}_{0}\left(\mathcal{U}_{0}(s, t)\right)+\sum_{q \geq 1} \mathcal{N}_{q}\left(\mathscr{U}_{0}, \mathscr{U}_{1}, \cdots, \mathscr{U}_{q}\right) \wp^{q}$.

Here, $\mathscr{U}_{q}$ for $q \geq 0$ is the set of linear equations with linear boundary conditions, which can be solved very easily.

The series in Equation (13) depends on $C_{1}, C_{2}, \cdots$. If it is convergent at $\wp=1$, then:

$$
\tilde{\mathscr{U}}\left(s, t ; C_{p}\right)=\mathscr{U}_{0}(s, t)+\sum_{q=1}^{m} \mathscr{U}_{q}\left(s, t, C_{p}\right) .
$$

Putting Equation (18) in Equation (8), one can obtain the residual expression in the form:

$$
\mathrm{R}\left(s, t ; C_{p}\right)=\mathscr{L}\left(\tilde{\mathscr{U}}\left(s, t ; C_{p}\right)\right)+\mathcal{N}\left(\tilde{\mathcal{U}}\left(s, t ; C_{p}\right)\right)+\hbar(s, t) .
$$

If $\mathrm{R}\left(s, t ; C_{p}\right)=0$, then $\tilde{\mathscr{U}}\left(s, t ; C_{p}\right)$ will be the exact solution. But generally, it is not possible in nonlinear problems.

For calculating the $C_{p}, p=1,2, \cdots, m$, one can utilize the least square technique as given by:

$$
\Phi\left(C_{p}\right)=\int_{0}^{t} \int_{r} R^{2}\left(s, t ; C_{p}\right) d s d t,
$$

where $R$ is the residual given by Equation (19) and

$$
\frac{\partial \Phi}{\partial C_{1}}=\frac{\partial \Phi}{\partial C_{2}}=\frac{\partial \Phi}{\partial C_{3}}=\cdots=\frac{\partial \Phi}{\partial C_{m}}=0 .
$$

The convergence based on $C_{1}, C_{2}, C_{3}, \cdots$ can be identified and minimized optimally by Equation (21).

\section{Numerical Examples}

In this section, the fractional OHAM is utilized to get the solution of time-fractional Navier-Stokes equations.

Example 1. Suppose a time-fractional Navier-Stokes equation:

$$
\frac{\partial^{\alpha} \mathscr{U}(s, t)}{\partial t^{\alpha}}=P+\frac{\partial^{2} \mathscr{U}(s, t)}{\partial s^{2}}+\frac{1}{s} \frac{\partial \mathcal{U}(s, t)}{\partial s},
$$

with the initial condition:

$$
\mathscr{U}(s, 0)=1-s^{2},
$$

where $\partial^{\alpha} / \partial t^{\alpha}$ is Caputo fractional derivative and $0<\alpha \leq 1$. The exact solution of Equation (22) is given by [14]:

$$
\mathcal{U}(s, t)=1-s^{2}+(P-4) t .
$$

According to Section 3, we can set up the homotopy in the following way:

$$
\begin{aligned}
(1-\wp) \frac{\partial^{\alpha} \omega(s, t ; \wp)}{\partial t^{\alpha}}= & \mathscr{H}\left(\wp, C_{p}\right) \\
& {\left[\frac{\partial^{\alpha} \omega(s, t ; \wp)}{\partial t^{\alpha}}-P-\frac{\partial^{2} \omega(s, t ; \wp)}{\partial s^{2}}-\frac{1}{s} \frac{\partial \omega(s, t ; \wp)}{\partial s}\right], }
\end{aligned}
$$

where

$$
\omega(s, t ; \wp)=\mathscr{U}_{0}(s, t)+\sum_{q=1}^{\infty} \mathcal{U}_{q}\left(s, t ; C_{p}\right) \wp^{q}, \quad p=1,2,3 \cdots,
$$

$$
\mathscr{H}\left(\wp, C_{p}\right)=\wp C_{1}+\wp^{2} C_{2}+\wp^{3} C_{3}+\wp^{4} C_{4}+\cdots .
$$

Plugging Equations (26) and (27) in (25) and equating the coefficient of the same powers of $\wp$, one can get the simpler problems, given as:

Zero-order problem:

$$
\frac{\partial^{\alpha} \mathscr{U}_{0}(s, t)}{\partial t^{\alpha}}=0, \quad \mathscr{U}_{0}(s, 0)=1-s^{2} .
$$

First-order problem:

$$
\begin{aligned}
\frac{\partial^{\alpha} \mathcal{U}_{1}\left(s, t ; C_{1}\right)}{\partial t^{\alpha}}= & \left(1+C_{1}\right) \frac{\partial^{\alpha} \mathcal{U}_{0}(s, t)}{\partial t^{\alpha}}-C_{1} P-C_{1} \frac{\partial^{2} \mathcal{U}_{0}(s, t)}{\partial s^{2}} \\
& -\frac{C_{1}}{s} \frac{\partial \mathcal{U}_{0}(s, t)}{\partial s}, \quad \mathscr{U}_{1}(s, 0)=0 .
\end{aligned}
$$

Respective solutions of Equations (28) and (29) after apply fractional integral and initial condition are given:

$$
\begin{aligned}
\mathcal{U}_{0}(s, t) & =1-s^{2}, \\
\mathcal{U}_{1}\left(s, t ; C_{1}\right) & =-\frac{C_{1}(P-4) t^{\alpha}}{\Gamma(1+\alpha)} .
\end{aligned}
$$

One can get the following expression:

$$
\begin{aligned}
\tilde{\mathscr{U}}(s, t) & =\mathscr{U}_{0}(s, t)+\mathscr{U}_{1}\left(s, t ; C_{1}\right)+\mathscr{U}_{2}\left(s, t ; C_{1}, C_{2}\right)+\cdots \\
& =1-s^{2}-\frac{C_{1}(P-4) t^{\alpha}}{\Gamma(1+\alpha)} .
\end{aligned}
$$

We used the least square method after finding the residual and then got the auxiliary constant value for $\alpha=1$; we have $C_{1}=-1$. Putting the value of $C_{1}$ in Equation (31), we get

$$
\tilde{U}(s, t)=1-s^{2}+\frac{(P-4) t^{\alpha}}{\Gamma(1+\alpha)} .
$$




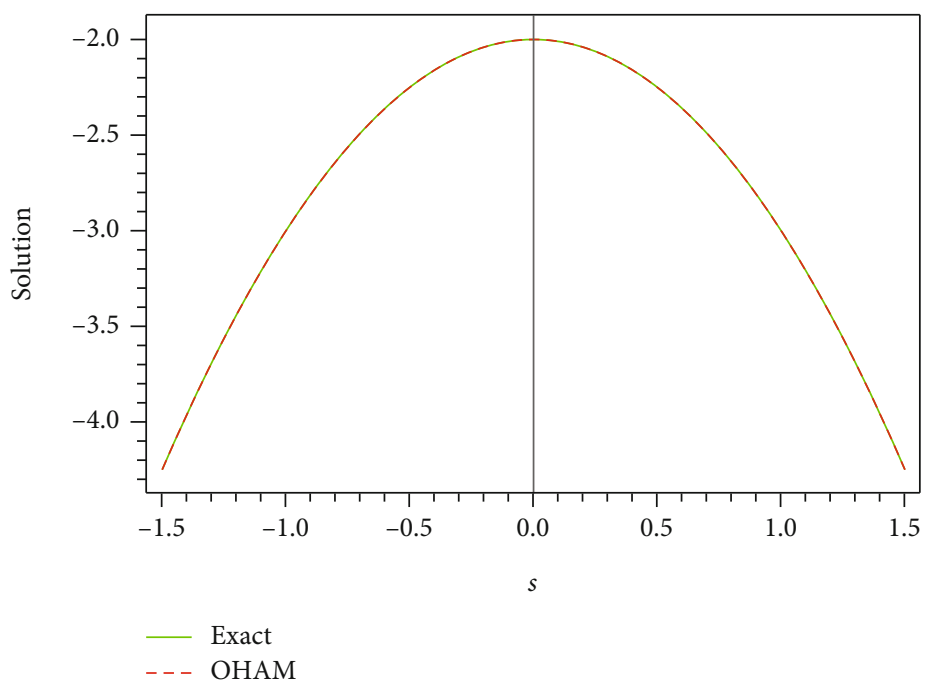

(a) Exact vs. OHAM solution



(b) Solution behavior for different values of $\alpha$

Figure 1: Continued. 


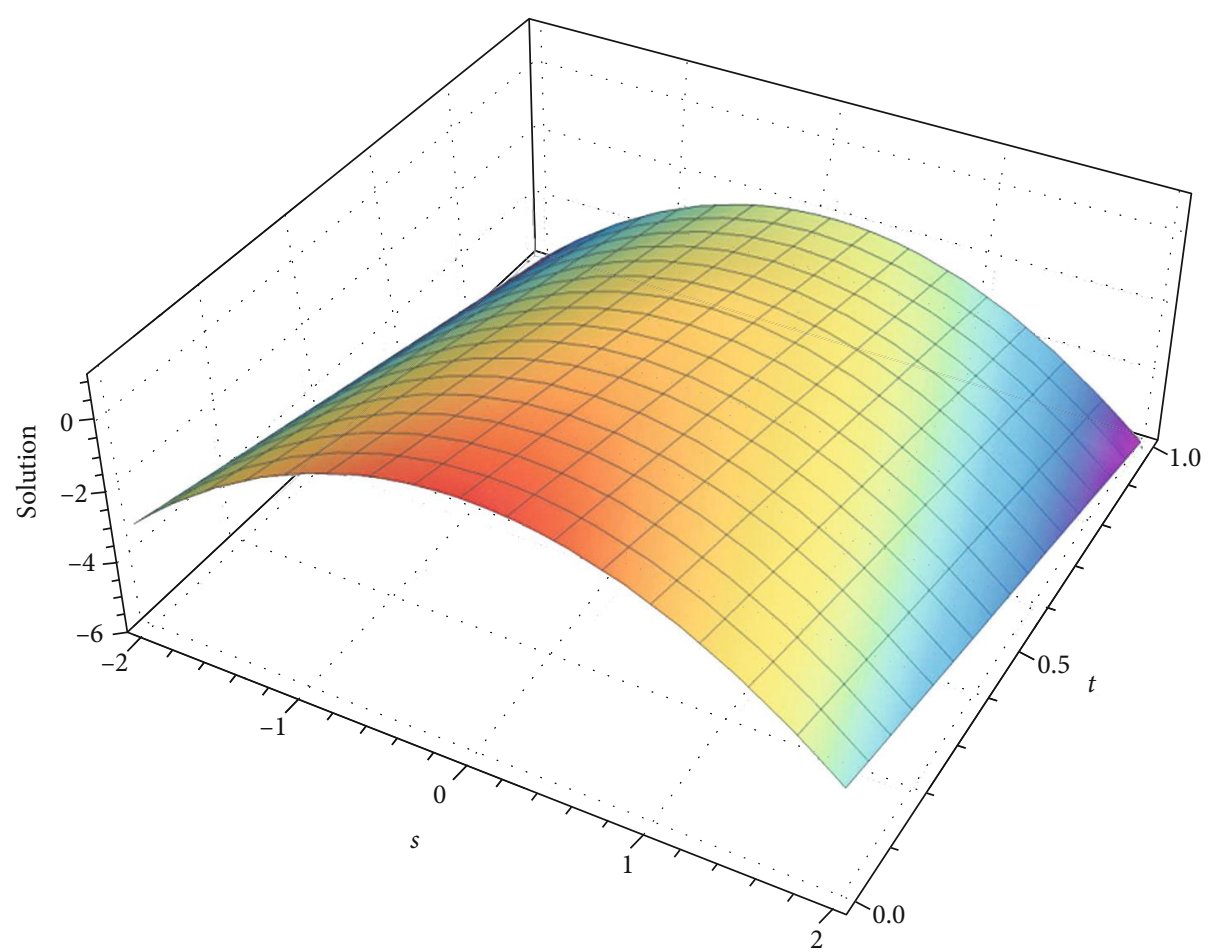

(c) Exact solution

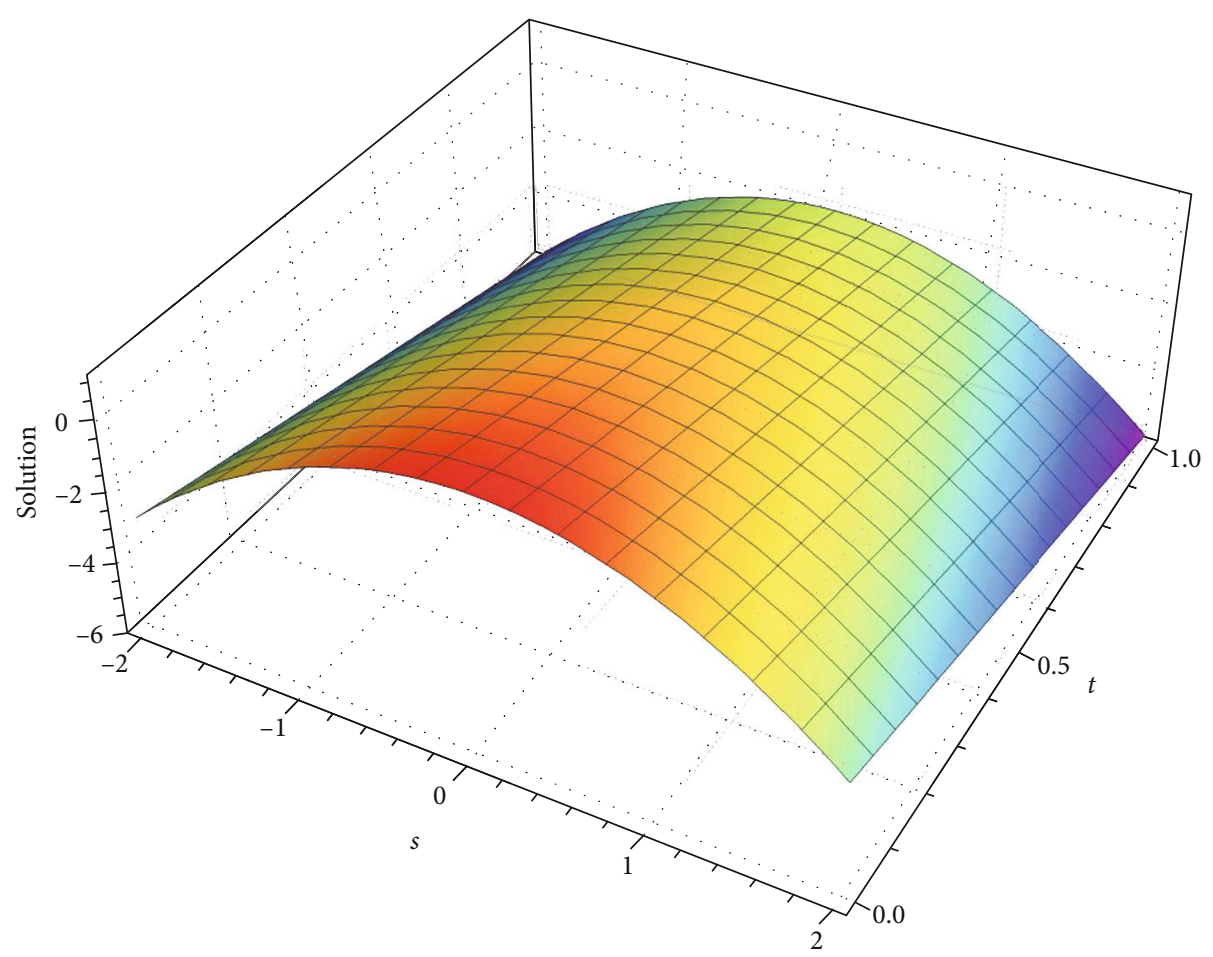

(d) OHAM solution for $\alpha=1$

Figure 1: Continued. 


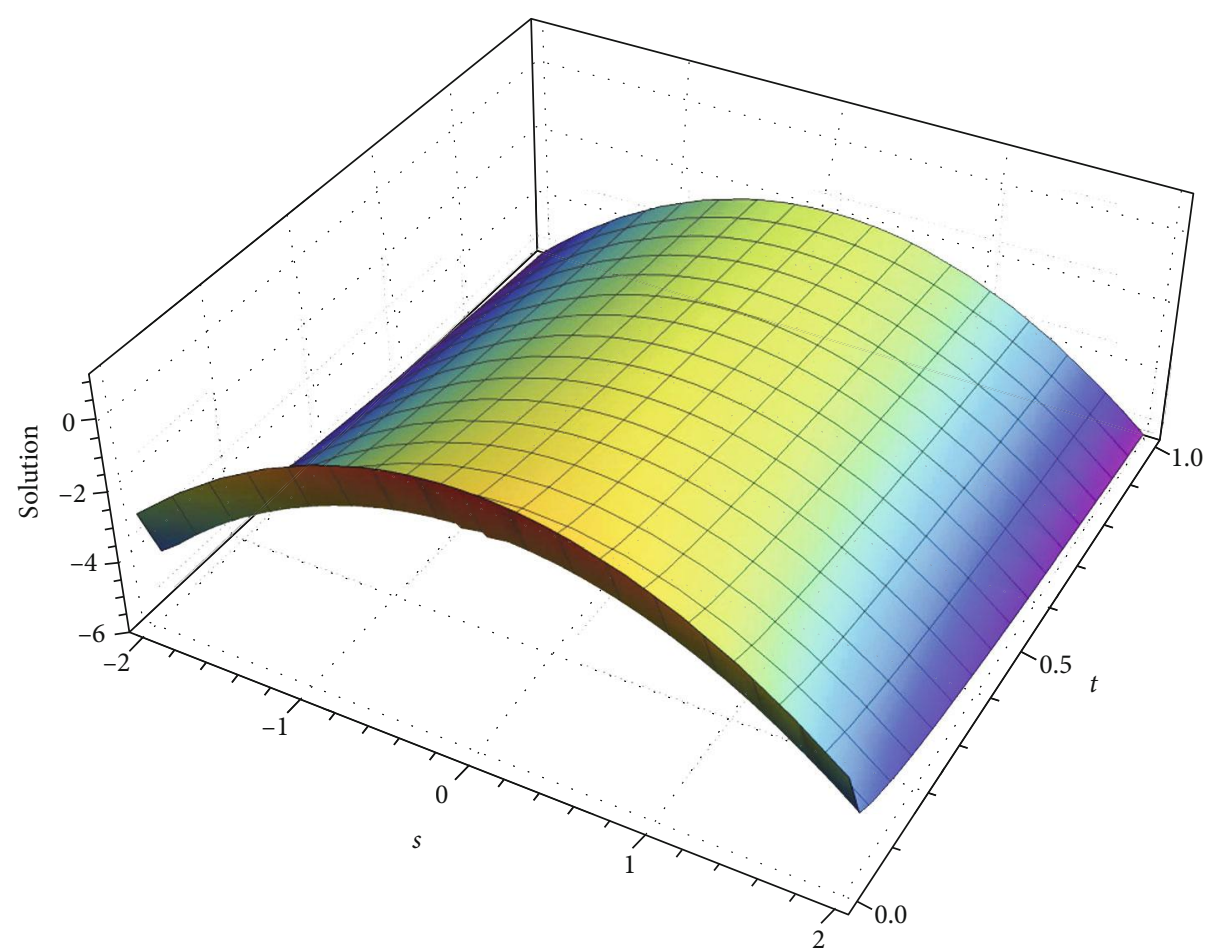

(e) Solution for $\alpha=0.2$

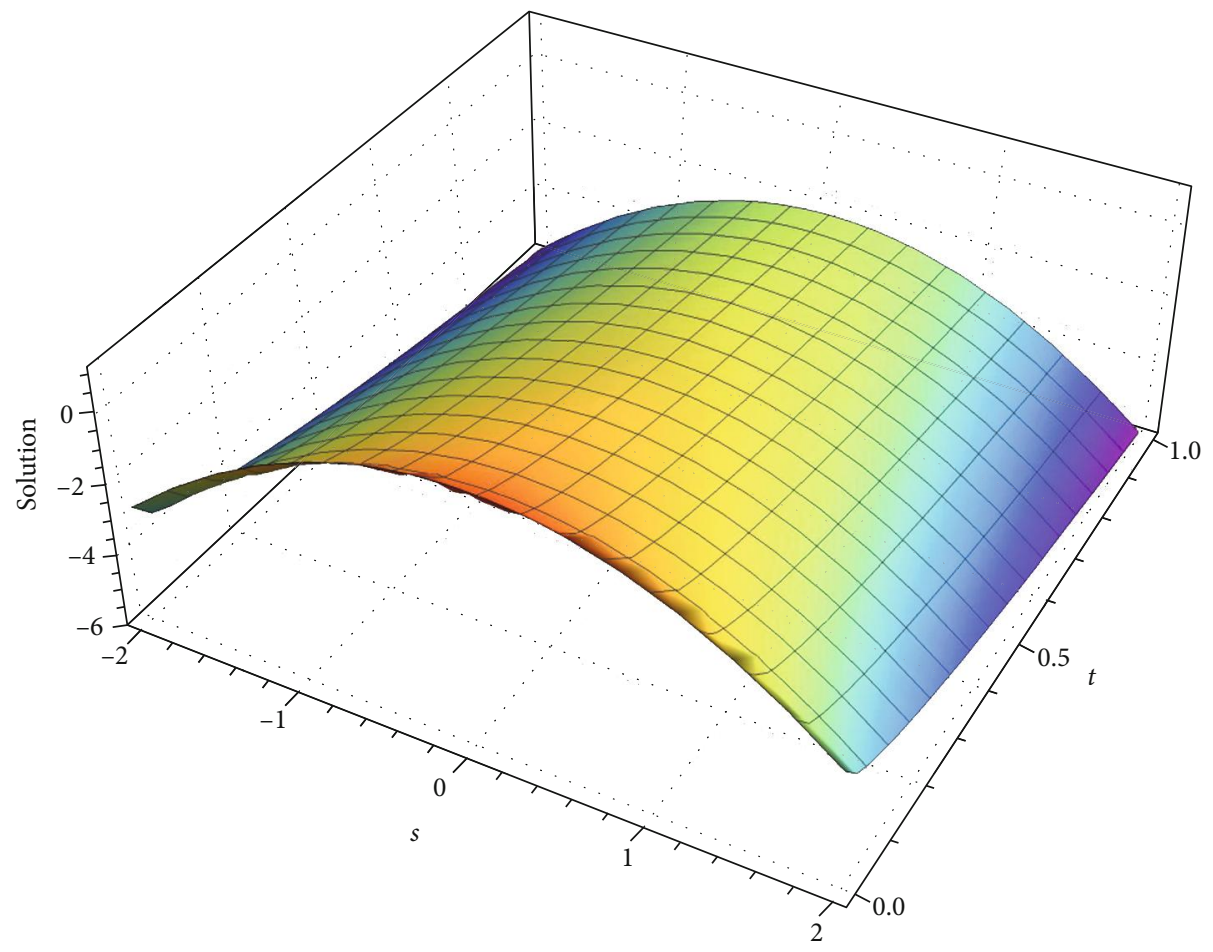

(f) Solution for $\alpha=0.5$

Figure 1: Exact solution and OHAM solution behavior of Example 1.

The result (32) is in complete agreement with [14, 25-31]. Figure 1 shows the evaluation results of the semianalytical solution for Example 1 when $P=1$ and show the dynamics of the obtained solution by OHAM for various noninteger order Brownian motions and for standard motions, i.e., for $\alpha=1$. It can be seen that the solution acquired via the above-mentioned technique is decreasing very swiftly with the increase in $t$ in Example 1, which is illustrated in Figure 1(b). Figure 1(a) shows the efficiency of the abovementioned method. Figures $1(\mathrm{e})$ and $1(\mathrm{f})$ show the solution behavior for $\alpha=0.2$ and $\alpha=0.5$. Besides, we have obtained the exact solution by OHAM. 
Example 2. Suppose a time-fractional Navier-Stokes equation:

$$
\frac{\partial^{\alpha} \mathcal{U}(s, t)}{\partial t^{\alpha}}=\frac{\partial^{2} \mathcal{U}(s, t)}{\partial s^{2}}+\frac{1}{s} \frac{\partial \mathcal{U}(s, t)}{\partial s},
$$

with the initial condition

$$
\mathcal{U}(s, 0)=s,
$$

where $\partial^{\alpha} / \partial t^{\alpha}$ is Caputo fractional derivative and $0<\alpha \leq 1$. We consider the first four terms of the exact solution of (33) from [14] is given by:

$$
\mathcal{U}(s, t)=s+\frac{t}{s}+\frac{t^{2}}{2 s^{3}}+\frac{9 t^{3}}{6 s^{5}}
$$

According to Section 3, we can set up the homotopy in the following way:

$$
(1-\wp) \frac{\partial^{\alpha} \omega(s, t ; \wp)}{\partial t^{\alpha}}=\mathscr{H}\left(\wp, C_{p}\right)\left[\frac{\partial^{\alpha} \omega(s, t ; \wp)}{\partial t^{\alpha}}-\frac{\partial^{2} \omega(s, t ; \wp)}{\partial s^{2}}-\frac{1}{s} \frac{\partial \omega(s, t ; \wp)}{\partial s}\right] \text {, }
$$

where

$$
\begin{aligned}
& \omega(s, t ; \wp)=\mathscr{U}_{0}(s, t)+\sum_{q=1}^{\infty} \mathcal{u}_{q}\left(s, t ; C_{p}\right) \wp^{q}, \quad p=1,2,3 \cdots \\
& \mathscr{H}\left(\wp, C_{p}\right)=\wp C_{1}+\wp^{2} C_{2}+\wp^{3} C_{3}+\wp^{4} C_{4}+\cdots
\end{aligned}
$$

Plugging Equations (37) and (38) in (36) and equating the coefficient of the same powers of $\wp$, one can get the simpler problems, given as:

Zero-order problem:

$$
\frac{\partial^{\alpha} \mathcal{U}_{0}(s, t)}{\partial t^{\alpha}}=0, \quad \mathcal{U}_{0}(s, 0)=s .
$$

First-order problem:

$$
\begin{aligned}
\frac{\partial^{\alpha} \mathcal{U}_{1}\left(s, t ; C_{1}\right)}{\partial t^{\alpha}}= & \left(1+C_{1}\right) \frac{\partial^{\alpha} \mathcal{U}_{0}(s, t)}{\partial t^{\alpha}}-C_{1} \frac{\partial^{2} \mathscr{U}_{0}(s, t)}{\partial s^{2}} \\
& -\frac{C_{1}}{s} \frac{\partial \mathcal{U}_{0}(s, t)}{\partial s}, \quad \mathscr{U}_{1}(s, 0)=0 .
\end{aligned}
$$

Second-order problem:

$$
\begin{aligned}
\frac{\partial^{\alpha} \mathscr{U}_{2}\left(s, t ; C_{1}, C_{2}\right)}{\partial t^{\alpha}}= & \left(1+C_{1}\right) \frac{\partial^{\alpha} \mathscr{U}_{1}\left(s, t ; C_{1}\right)}{\partial t^{\alpha}}-C_{1} \frac{\partial^{2} \mathcal{U}_{1}\left(s, t ; C_{1}\right)}{\partial s^{2}} \\
& -\frac{C_{1}}{s} \frac{\partial \mathcal{U}_{1}\left(s, t ; C_{1}\right)}{\partial s}+C_{2} \frac{\partial^{\alpha} \mathcal{U}_{0}(s, t)}{\partial t^{\alpha}} \\
& -C_{2} \frac{\partial^{2} \mathcal{U}_{0}(s, t)}{\partial s^{2}}-\frac{C_{2}}{s} \frac{\partial \mathcal{U}_{0}(s, t)}{\partial s}, \quad \mathscr{U}_{2}(s, 0)=0 .
\end{aligned}
$$

TABle 1: $C_{1}, C_{2}, C_{3}$ for various values of $\alpha$.

\begin{tabular}{lccc}
\hline$\alpha$ & $C_{1}$ & $C_{2}$ & $C_{3}$ \\
\hline 0.7 & -1.036692817879 & -0.001240278601 & $-4.24687 \times 10^{-5}$ \\
0.8 & -1.020447062606 & -0.000376864638 & $-7.02931 \times 10^{-6}$ \\
0.9 & -1.010550021386 & -0.000101578844 & $-9.88500 \times 10^{-7}$ \\
0.10 & -0.999953117565 & -0.000040256679 & $3.30467 \times 10^{-8}$ \\
\hline
\end{tabular}

TABLE 2: Comparison of exact and OHAM solution.

\begin{tabular}{lccc}
\hline$s$ & Exact & OHAM & Abs error \\
\hline 1.0 & 2.94735999 & 2.94736040 & $4.04145499 \times 10^{-7}$ \\
1.2 & 3.05262208 & 3.05262204 & $3.73698401 \times 10^{-8}$ \\
1.4 & 3.16486784 & 3.16486694 & $8.94947017 \times 10^{-7}$ \\
1.6 & 3.28483456 & 3.28483228 & $2.27227761 \times 10^{-6}$ \\
1.8 & 3.41325952 & 3.41325524 & $4.27305321 \times 10^{-6}$ \\
2.0 & 3.55088000 & 3.55087299 & $7.00096540 \times 10^{-6}$ \\
\hline
\end{tabular}

Third-order problem:

$$
\begin{aligned}
\frac{\partial^{\alpha} \mathcal{U}_{3}\left(s, t ; C_{1}, C_{2}, C_{3}\right)}{\partial t^{\alpha}}= & \left(1+C_{1}\right) \frac{\partial^{\alpha} \mathcal{U}_{2}\left(s, t ; C_{1}, C_{2}\right)}{\partial t^{\alpha}}-C_{1} \frac{\partial^{2} \mathcal{U}_{2}\left(s, t ; C_{1}, C_{2}\right)}{\partial s^{2}} \\
& -\frac{C_{1}}{s} \frac{\partial \mathcal{U}_{2}\left(s, t ; C_{1}, C_{2}\right)}{\partial s}+C_{2} \frac{\partial^{\alpha} \mathscr{U}_{1}\left(s, t ; C_{1}\right)}{\partial t^{\alpha}} \\
& -C_{2} \frac{\partial^{2} \mathcal{U}_{1}\left(s, t ; C_{1}\right)}{\partial s^{2}}-\frac{C_{2}}{s} \frac{\partial \mathcal{U}_{1}\left(s, t ; C_{1}\right)}{\partial s} \\
& +C_{3} \frac{\partial^{\alpha} \mathcal{U}_{0}(s, t)}{\partial t^{\alpha}}-C_{3} \frac{\partial^{2} \mathscr{U}_{0}(s, t)}{\partial s^{2}} \\
& -\frac{C_{3}}{s} \frac{\partial \mathcal{U}_{0}(s, t)}{\partial s}, \mathscr{U}_{3}(s, 0)=0 .
\end{aligned}
$$

Respective solutions of Equations (39)-(42) after apply fractional integral and initial condition are given:

$$
\begin{aligned}
\mathscr{U}_{0}(s, t) & =s \\
\mathscr{U}_{1}\left(s, t ; C_{1}\right) & =-\frac{C_{1} t^{\alpha}}{s \Gamma(1+\alpha)}, \\
\mathcal{U}_{2}\left(s, t ; C_{1}, C_{2}\right)= & -\frac{\left(C_{1}+C_{1}^{2}+C_{2}\right) t^{\alpha}}{s \Gamma(1+\alpha)}+\frac{C_{1}^{2} t^{2 \alpha}}{s^{3} \Gamma(1+2 \alpha)}, \\
\mathscr{U}_{3}\left(s, t ; C_{1}, C_{2}, C_{3}\right)= & -\frac{\left(C_{1}+2 C_{1}^{2}+C_{1}^{3}+2 C_{1} C_{2}+C_{2}+C_{3}\right)(t)^{\alpha}}{s \Gamma(1+\alpha)} \\
& +\frac{2 C_{1}\left(C_{1}+C_{1}^{2}+C_{2}\right) t^{2 \alpha}}{s^{3} \Gamma(1+2 \alpha)}-\frac{9 C_{1}^{3} t^{3 \alpha}}{s^{5} \Gamma(1+3 \alpha)} . \\
\vdots &
\end{aligned}
$$

One can calculate the next order problem solutions by above similar process. In the end, we can get the expression: 


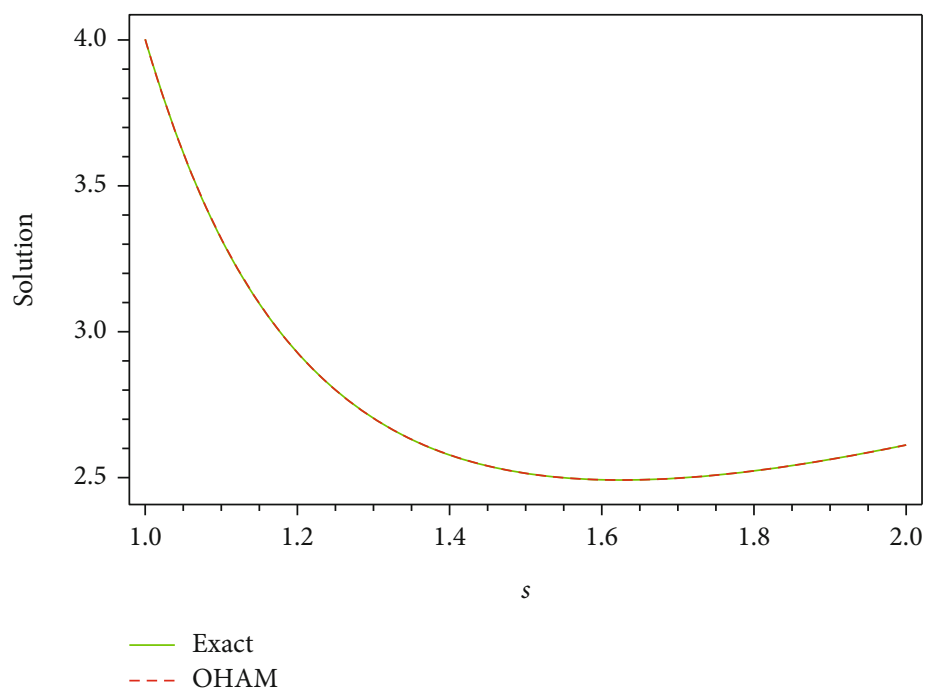

(a) Exact vs. OHAM solution

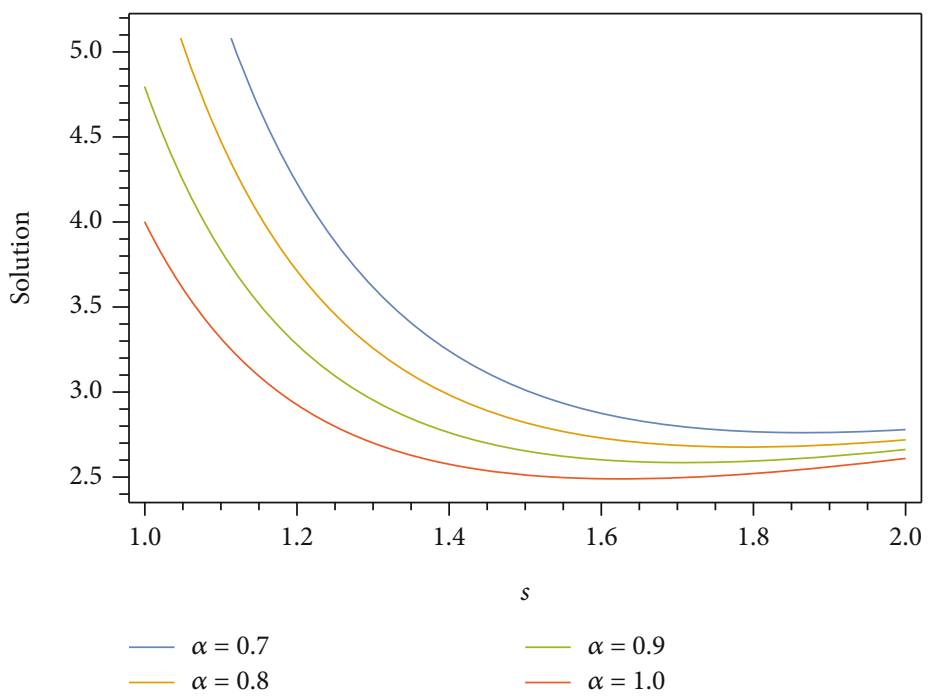

(b) Solution behavior for different values of $\alpha$

Figure 2: Continued. 


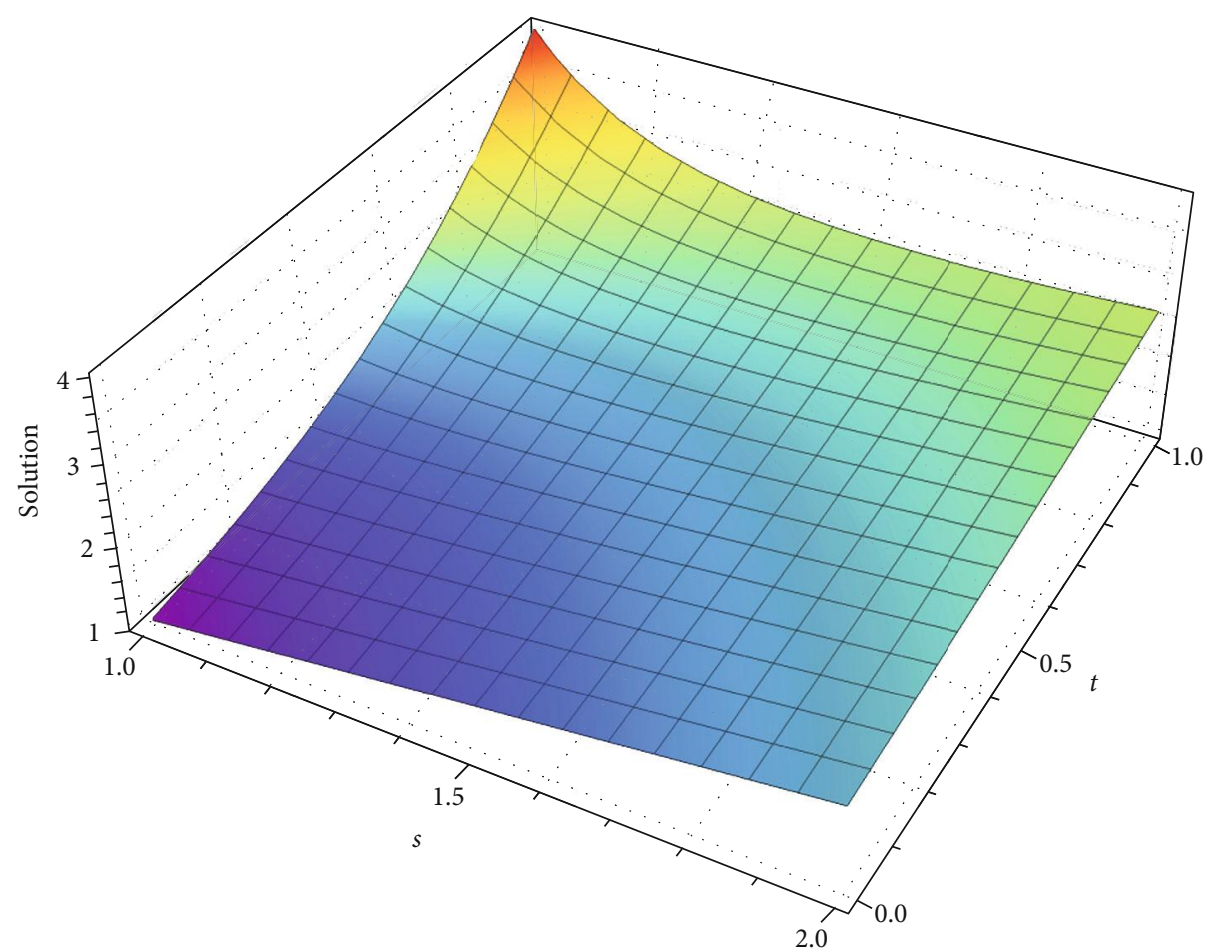

(c) Exact solution

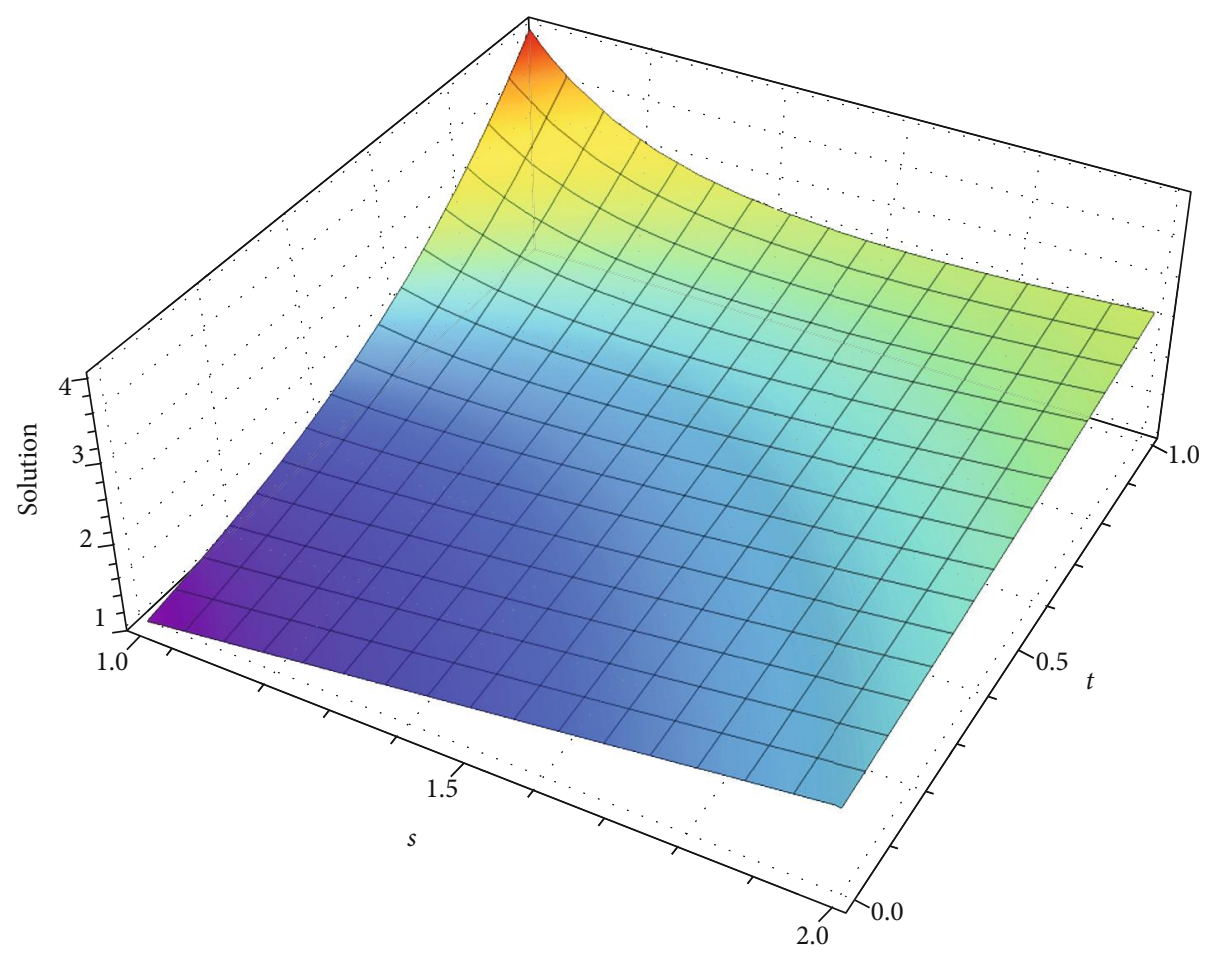

(d) OHAM solution for $\alpha=1$

Figure 2: Continued. 


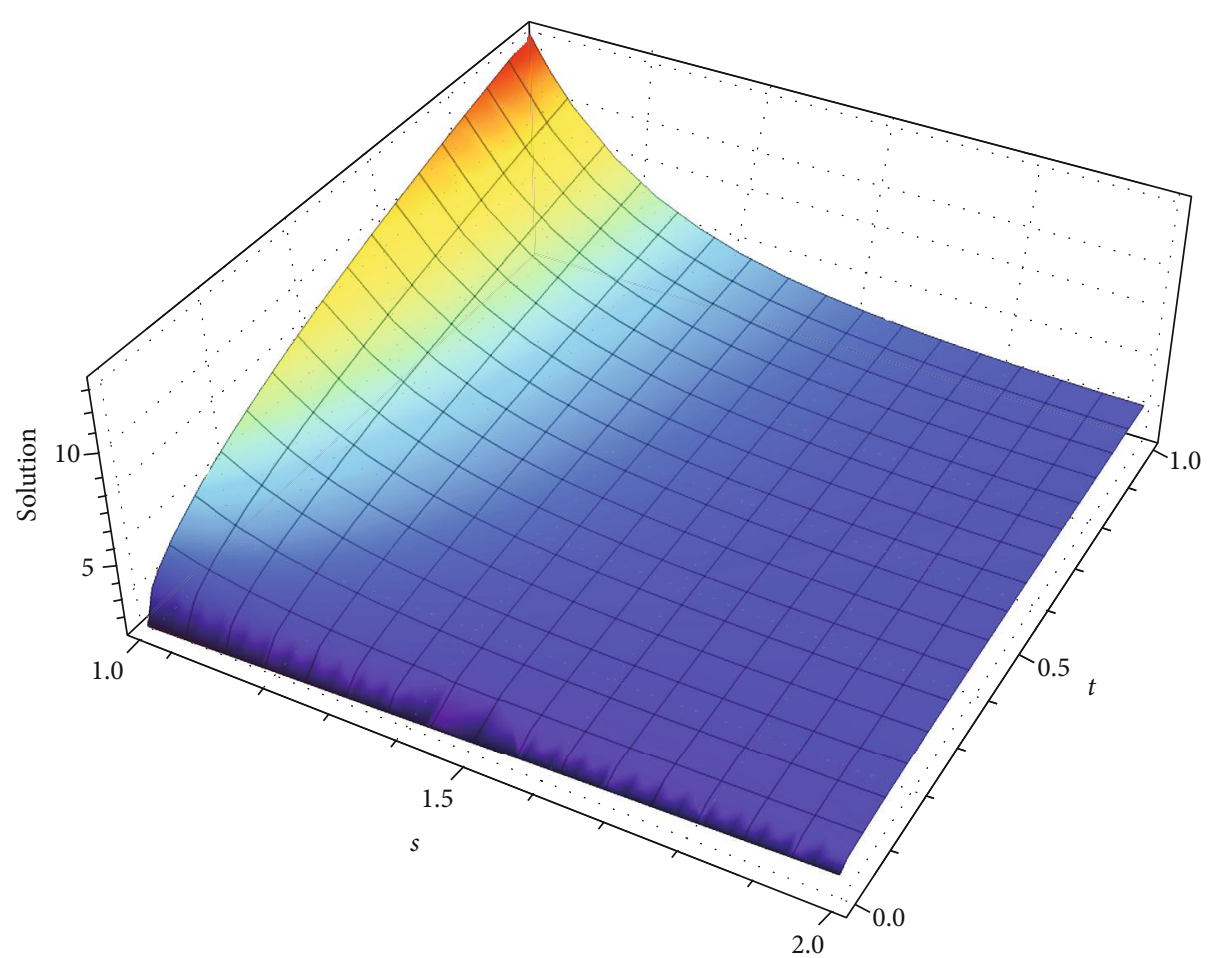

(e) Solution for $\alpha=0.2$

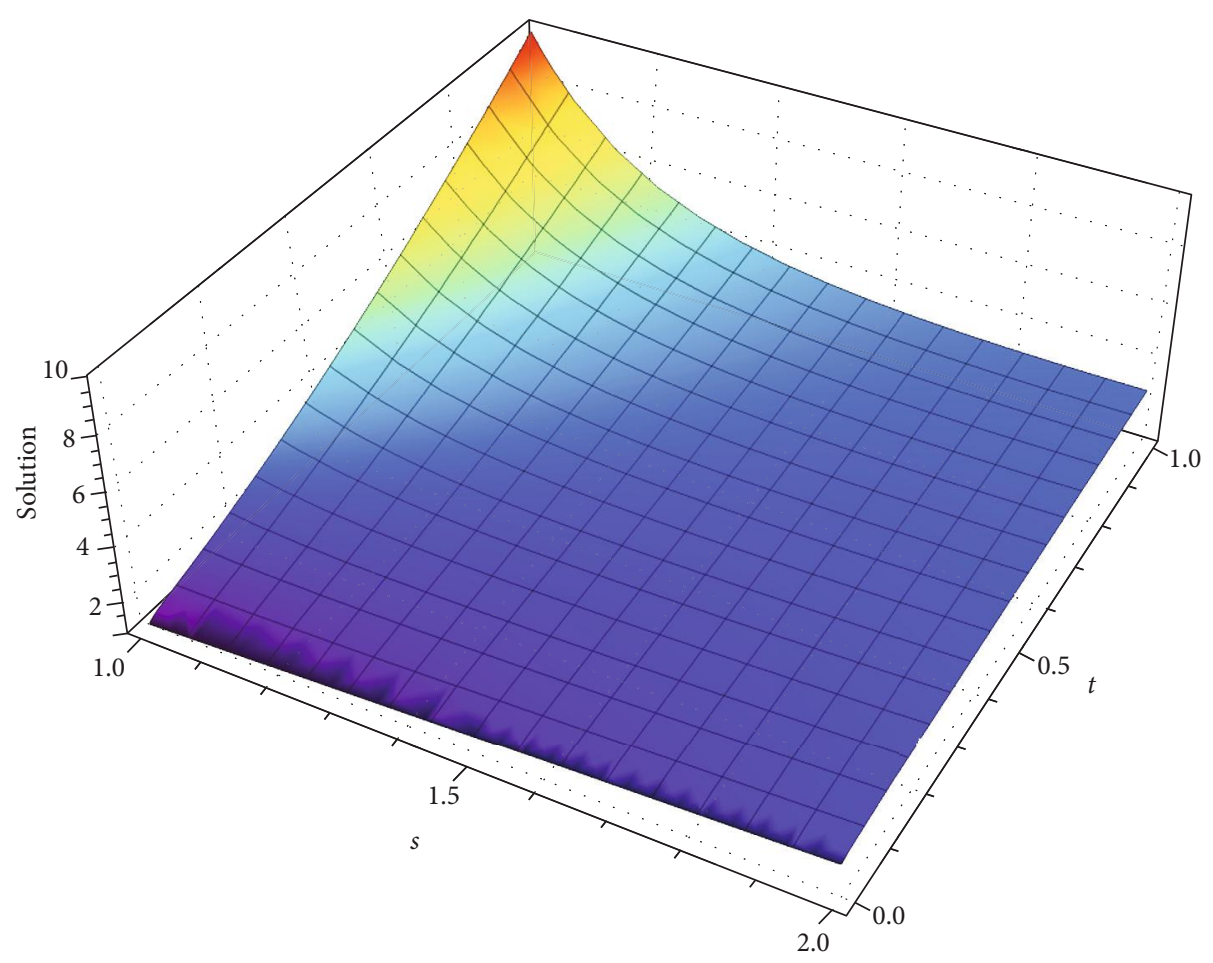

(f) Solution for $\alpha=0.5$

FIGURE 2: Exact solution and OHAM solution behavior of Example 2. 


$$
\begin{aligned}
\tilde{\mathcal{U}}(s, t)= & \mathcal{U}_{0}(s, t)+\mathcal{U}_{1}\left(s, t ; C_{1}\right)+\mathcal{U}_{2}\left(s, t ; C_{1}, C_{2}\right) \\
& +\mathcal{U}_{3}\left(s, t ; C_{1}, C_{2}, C_{3}\right)+\cdots \\
= & s-\frac{\left(3 C_{1}+3 C_{1}^{2}+C_{1}^{3}+2 C_{2}+2 C_{1} C_{2}+C_{3}\right) t^{\alpha}}{s \Gamma(1+\alpha)} \\
& +\frac{C_{1}\left(3 C_{1}+2 C_{1}^{2}+2 C_{2}\right) t^{2 \alpha}}{s^{3} \Gamma(1+2 \alpha)}-\frac{9 C_{1}^{3} t^{3 \alpha}}{s^{5} \Gamma(1+3 \alpha)} \cdots .
\end{aligned}
$$

We used the least square method after finding the residual and then got the auxiliary constant value for $\alpha$ (see Table 1). The absolute error of both solutions can be seen in Table 2.

Putting the values of $C_{1}, C_{2}$, and $C_{3}$ for $\alpha=1$ in Equation (44), we get

$$
\tilde{\mathscr{U}}(s, t)=s+\frac{t^{\alpha}}{s \Gamma(1+\alpha)}+\frac{1.00008 t^{2 \alpha}}{s^{3} \Gamma(1+2 \alpha)}+\frac{8.99873 t^{3 \alpha}}{s^{5} \Gamma(1+3 \alpha)}
$$

Figure 2 shows the solution behavior of the time-fractional order Navier-Stokes equation by OHAM. In Figure 2(a), the solution curves are decreasing rapidly for higher fractional orders until we get the standard motion of fluid for $\alpha=1$, and Figure 2(b) shows the proposed method's effectiveness and reliability. Figures 2(e) and 2(f) show the OHAM solution behavior for $\alpha=0.2$ and $\alpha=0.5$, respectively. The obtained results reveal that the method mentioned above is an efficient tool to study such types of fractional order fluid mechanics problems, which can be seen in Table 2 .

\section{Conclusion}

In this study, fractional-order OHAM is successfully implemented to obtain the optimal solutions of timefractional Navier-Stokes equation. From the acquired results, it can be seen that OHAM is an efficient and reliable semianalytical technique to approximate the solution of different fractional-order linear and nonlinear problems appearing in engineering and science. The above-mentioned technique provides a simple approach to control and adjust the convergence of the series solution utilizing the constants $C_{p}$ 's which are determined optimally. Two examples have been studied to illustrate the efficiency and versatility of this approach. The OHAM solution of the first example is the same as the exact solution, and for the OHAM solution of the second example, the obtained numerical approximation of the solution has a strong agreement with the exact solution. Besides, when the order of approximation increases, the error accuracy of the numerical solution decreases and becomes closer to the exact solutions. The proposed technique's fast accuracy and convergence are valid reasons for the researcher to use it for various problems in science and technology. It has been noted that the semianalytical solutions by extended formulation are in remarkable agreement with the exact solutions.

\section{Data Availability}

No real data were used to support this study.

\section{Conflicts of Interest}

This work does not have any conflicts of interest.

\section{Authors' Contributions}

The authors have contributed equally to this manuscript.

\section{Acknowledgments}

This research is studied at the School of Engineering, Monash University Malaysia.

\section{References}

[1] S. R. Bistafa, "On the development of the Navier-Stokes equation by Navier," Revista Brasileira de Ensino de Física, vol. 40, no. 2, p. 12, 2018.

[2] R. Hilfer, Applications of Fractional Calculus in Physics, World Scientific, Singapore, 2000.

[3] S. Das, Functional Fractional Calculus, Springer Berlin Heidelberg, Berlin, Heidelberg, 2011.

[4] K. Oldham, "Fractional differential equations in electrochemistry," Advances in Engineering Software, vol. 41, no. 1, pp. 9-12, 2010.

[5] V. E. Tarasov, Fractional Dynamics: Applications of Fractional Calculus to Dynamics of Particles, Fields and Media, Springer Science \& Business Media, 2011.

[6] R. Matušu, "Application of fractional order calculus to control theory," International Journal of Mathematical Models and Methods in Applied Sciences, vol. 5, no. 7, pp. 1162-1169, 2011.

[7] R. P. Meilanov and R. A. Magomedov, "Thermodynamics in fractional calculus," Journal of Engineering physics and thermophysics, vol. 87, no. 6, pp. 1521-1531, 2014.

[8] F. Meral, T. Royston, and R. Magin, "Fractional calculus in viscoelasticity: an experimental study," Communications in Nonlinear Science and Numerical Simulation, vol. 15, no. 4, pp. 939-945, 2010.

[9] O. W. Abdulwahhab and N. H. Abbas, "A new method to tune a fractional-order PID controller for a twin rotor aerodynamic system," Arabian Journal for Science and Engineering, vol. 42, no. 12, pp. 5179-5189, 2017.

[10] Y. Jiang, B. Zhang, X. Shu, and Z. Wei, "Fractional-order autonomous circuits with order larger than one," Journal of Advanced Research, vol. 25, pp. 217-225, 2020.

[11] F. Liu and K. Burrage, "Novel techniques in parameter estimation for fractional dynamical models arising from biological systems," Computers \& Mathematcs with Applications, vol. 62, no. 3, pp. 822-833, 2011.

[12] N. Ali Shah, D. Vieru, and C. Fetecau, "Effects of the fractional order and magnetic field on the blood flow in cylindrical domains," Journal of Magnetism and Magnetic Materials, vol. 409, pp. 10-19, 2016.

[13] N. H. Can, H. Jafari, and M. N. Ncube, "Fractional calculus in data fitting," Alexandria Engineering Journal, vol. 59, no. 5, pp. 3269-3274, 2020. 
[14] S. Momani and Z. Odibat, "Analytical solution of a timefractional Navier-Stokes equation by Adomian decomposition method," Applied Mathematics and Computation, vol. 177, no. 2, pp. 488-494, 2006.

[15] S. J. Liao, On the Proposed Homotopy Analysis Technique for Nonlinear Problems and its Applications, [Ph.D. thesis], Shanghai Jio Tong University, Shanghai, China, 1992.

[16] J. He, "An approximate solution technique depending on an artificial parameter: a special example," Communications in Nonlinear Science and Numerical Simulation, vol. 3, no. 2, pp. 92-97, 1998.

[17] D. D. Ganji and M. Rafei, "Solitary wave solutions for a generalized Hirota-Satsuma coupled KdV equation by homotopy perturbation method," Physics Letters A, vol. 356, no. 2, pp. 131-137, 2006.

[18] D. Lu and J. Liu, "Application of the homotopy analysis method for solving the variable coefficient KdV-Burgers equation," Abstract and Applied Analysis, vol. 2014, Article ID 309420, 4 pages, 2014.

[19] A. M. Siddiqui, S. Irum, and A. R. Ansari, "A solution of the unsteady squeezing flow of a viscous fluid between parallel plates using the homotopy perturbation method," Mathematical Modelling and Analysis, vol. 13, no. 4, pp. 565-576, 2008.

[20] V. Marinca and N. Herisanu, "Application of optimal homotopy asymptotic method for solving nonlinear equations arising in heat transfer," International Communications in Heat and Mass Transfer, vol. 35, no. 6, pp. 710-715, 2008.

[21] N. Herisanu, V. Marinca, T. Dordea, and G. Madescu, "A new analytical approach to nonlinear vibration of an electrical machine," Proceedings of the Romanian Academy, Series A, vol. 9, no. 3, pp. 229-236, 2008.

[22] V. Marinca and N. Herisanu, "Determination of periodic solutions for the motion of a particle on a rotating parabola by means of the optimal homotopy asymptotic method," Journal of Sound and Vibration, vol. 329, no. 9, pp. 1450-1459, 2010.

[23] N. Herisanu and V. Marinca, "Accurate analytical solutions to oscillators with discontinuities and fractional-power restoring force by means of the optimal homotopy asymptotic method," Computers \& Mathematics with Applications, vol. 60, no. 6, pp. 1607-1615, 2010.

[24] V. Marinca, N. Herişanu, C. Bota, and B. Marinca, "An optimal homotopy asymptotic method applied to the steady flow of a fourth- grade fluid past a porous plate," Applied Mathematics Letters, vol. 22, no. 2, pp. 245-251, 2009.

[25] S. Kumar, D. Kumar, S. Abbasbandy, and M. M. Rashidi, "Analytical solution of fractional Navier-Stokes equation by using modified Laplace decomposition method," Ain Shams Engineering Journal, vol. 5, no. 2, pp. 569-574, 2014.

[26] A. Prakash, D. G. Prakasha, and P. Veeresha, "A reliable algorithm for time-fractional Navier-Stokes equations via Laplace transform," Nonlinear Engineering, vol. 8, no. 1, pp. 695-701, 2019.

[27] D. Kumar, J. Singh, and S. Kumar, "A fractional model of Navier-Stokes equation arising in unsteady flow of a viscous fluid," Journal of the Association of Arab Universities for Basic and Applied Sciences, vol. 17, no. 1, pp. 14-19, 2015.

[28] K. Wang and S. Liu, "Analytical study of time-fractional Navier-Stokes equations by transform methods," Advances in Difference Equations, vol. 2016, no. 1, Article ID 61, 2016.
[29] S. Maitama, "Analytical solution of time-fractional NavierStokes equation by natural homotopy perturbation method," Progress in Fractional Differentiation and Applications, vol. 4, no. 2, pp. 123-131, 2018.

[30] B. K. Albuohimad, "Analytical technique of the fractional Navier-Stokes model by Elzaki transform and homotopy perturbation method," AIP Conference Proceedings, vol. 2144, article 050002, 2019.

[31] N. A. Khan, A. Ara, S. A. Ali, and A. Mahmood, "Analytical study of Navier-Stokes equation with fractional orders using He's homotopy perturbation and variational iteration methods," International Journal of Nonlinear Sciences and Numerical Simulation, vol. 10, no. 9, pp. 1127-1134, 2009.

[32] A. A. Kilbas, H. M. Srivastava, and J. J. Trujillo, Theory and Applications of Fractional Differential Equations, NorthHolland Mathematics Studies, vol. 204, Elsevier, Amsterdam, 2006.

[33] S. Iqbal, M. Idrees, A. M. Siddiqui, and A. R. Ansari, "Some solutions of the linear and nonlinear Klein-Gordon equations using the optimal homotopy asymptotic method," Applied Mathematics and Computation, vol. 216, no. 10, pp. 28982909, 2010. 